\title{
Editorial \\ Kompetenz und Performanz in der Bildungsforschung
}

\section{Werner Wicki}

Die Schweizerische Gesellschaft für Bildungsforschung (SGBF) beschäftigte sich an ihrem Jahreskongress 2014 in Luzern mit dem Thema «Kompetenz und Performanz». Dieses Thema war naheliegend, denn Kompetenz wurde in den letzten 10-15 Jahren zu einem «Mega»-Begriff, was nicht zuletzt damit zu tun hat, dass seine "Anbändelungswut» enorm ist, wie Reichenbach (2014) nicht ohne Spott - festgestellt hat: Neben den bislang schon populären sozialen, personalen und fachlichen Kompetenzen, wobei sich die fachlichen weiter aufschlüsseln lassen in mathematische, sprachliche, fremdsprachliche, naturwissenschaftliche, musikalische etc., verweisen diverse Publikationen auf weitere Kompetenzen wie Selbstregulations-, Auftretens-, Methoden-, Partizipations-, Planungs-, Umsetzungs-, Diagnose-, transversale und interkulturelle Kompetenzen (z.B. De Haan, 2002; Schütte, Wirth \& Leutner, 2010; Audigier, 2015 in diesem Heft), um nur einige zu nennen.

Die Präsenz des Begriffs kann auch mit einfachen bibliometrischen Mitteln nachgewiesen werden, z.B. indem die jährliche Anzahl Publikationen mit «Kompetenz» (im Titel) im Zeitverlauf und im Vergleich mit anderen Begriffen (z.B. Lernen) analysiert wird. Führt man diese Analyse einerseits mittels FIS Bildung für die deutschsprachigen Publikationen (einmal für Kompetenz und einmal für Lernen) und zusätzlich mittels ERIC für die englischsprachigen Publikationen (diesmal mit "Competence» und «Learning») durch, so erhält man die in der Tabelle 1 dargestellten Verhältnisse.

Tabelle 1: Publikationen mit Kompetenz/Competence und Lernen/Learning 1995-2010

\begin{tabular}{|l|c|c|c|c|}
\hline \multirow{2}{*}{ Jahr } & \multicolumn{2}{|c|}{ ERIC } & \multicolumn{2}{c|}{ FIS Bildung } \\
\hline 1995 & Competence & Learning & Kompetenz & Lernen \\
\hline 2000 & 98 & 2329 & 37 & 590 \\
\hline 2005 & 96 & 3134 & 55 & 836 \\
\hline 2010 & 96 & 3239 & 99 & 874 \\
\hline
\end{tabular}


Im Vergleich zum relativen Boom der auf Kompetenzen bezogenen deutschsprachigen Publikationen bleibt der Publikationsumfang bei den englischsprachigen Arbeiten relativ zu andern Themen (z.B. Lernen) konstant auf bescheidenem Niveau, so dass man den Eindruck erhält, der Kompetenzdiskurs und die Erforschung der verschiedenen Kompetenzen sei ein europäisches Phänomen, das im Gefolge der PISA-Studien aufgekommen ist (Deutschland startete bekanntlich mit in der Presse skandalisierten mittelmässigen Ergebnissen) und dann eine Eigendynamik entwickelt hat, wie sie für viele wissenschaftliche Disziplinen typisch ist.

Der von der OECD (im Rahmen der PISA-Studien) angeregte Kompetenzbegriff zielt auf Fähigkeiten und Fertigkeiten, anhand derer das Individuum situativ bestimmte Problem- und Aufgabenstellungen lösen kann (vgl. auch Audigier, 2015, in diesem Heft). Nach dieser Auffassung genügt es z.B. nicht zu wissen, wie man den Inhalt eines Gefässes berechnet, vielmehr wird (bei entsprechender Kompetenz) erwartet, dass dieses Wissen im Rahmen einer praktischen Problemstellung (z.B. Eisenbahnwagen, der geladen wird) zielführend eingesetzt wird. «Kompetenzorientierung» meint deshalb tatsächlich etwas mehr als die Mutation der Lernziele der alten Lehrpläne zu «Können-Formulierungen» in den neuen Lehrplänen.

Anspruchsvoll wird der Kompetenzbegriff zusätzlich, wenn man - wie dies im Lehrplan 21 oder im PER (Plan d'études romand) der Fall ist - versucht, zu jeder Kompetenz auch sogenannte Kompetenzstufen zu definieren, die entwicklungslogisch aufeinander folgen. Bisherige Lehrpläne haben zwar solche Abfolgen implizit ebenfalls enthalten. Die Innovation des Lehrplans 21 oder des PER besteht jedoch darin, dass nun explizit eine Abfolge postuliert und vorgegeben wird. Dass diese Abfolge bis heute in den wenigsten Fällen empirisch überprüft ist und letztlich auf fachdidaktischem Erfahrungswissen basiert, ist eine Schwachstelle sowohl des Lehrplans 21 als auch des PER, die zu beheben ein Desiderat zukünftiger Lernforschung ist.

Man kann die enorme Arbeit, die beispielsweise beim Definieren der 363 Kompetenzen mit insgesamt 2304 Kompetenzstufen des Deutschschweizer Lehrplans 21 geleistet wurde, mit dem Hinweis auf deren geringe Wirksamkeit auf den Schulunterricht belächeln (Reichenbach, 2014) oder den ganzen Ansatz als gesellschaftliche Übersteuerung der Schule abtun und ablehnen (Herzog, 2013) - aber: 2304 Kompetenzstufen geteilt durch 11 Jahre sind durchschnittlich etwas mehr als 200 Kompetenzstufen pro Jahr geteilt durch ca. ein Dutzend Fächer und überfachliche Aspekte ergibt weniger als 20 Kompetenzstufen pro Jahr und Fach oder ca. alle 2 Wochen eine Kompetenzstufe pro Fach - eigentlich gut machbar, wenn man sich die Kompetenzstufenbeschreibungen konkret vor Augen führt (z.B. können Vielecke und gerade Prismen zur Berechnung von Flächeninhalten und Volumen zerlegen) und wenn man voraussetzt, dass Unterrichtszeit mehrheitlich Lernzeit ist. Letzteres bleibt auch mit den neuen Lehrplänen in der Verantwortung der Lehrperson. 
Nun entstehen ja die fachspezifischen Kompetenzformulierungen nicht im luftleeren Raum sondern vor dem Hintergrund eines Fachdiskurses wie ihn z.B. Audigier (2015, in diesem Heft) am Beispiel der Bildung für eine Nachhaltige Entwicklung (BNE) vorstellt. BNE gehört zu den sogenannten Bindestrichdisziplinen, die gesellschaftlich virulente Themen (wie Gesundheit, Umwelt, Sexualität, Medien etc.) aufgreifen, deren Inhalte - mit gutem Grund (de Haan 2002) - in die Schule drängen. Audigier plädiert für die Integration dieser Themen in bestehende Fächer, insbesondere in Geschichte, Geographie und politische Bildung (ein Weg, der z.B. auch im Lehrplan 21 beschritten wird).

Demeuse et al. (2015, in diesem Heft) beschreiben die je nach Stufe und Anspruchsniveau stark unterschiedlichen Curricula des stark segregierten Schulsystems in der Französischen Gemeinschaft Belgiens. Nicht nur sind dort die Curricula verschiedener Stufen schlecht bis gar nicht miteinander verknüpft, die oft stark unterspezifizierten Curricula variieren (in ihrer Ausgestaltung) gelegentlich sogar von Schule zu Schule innerhalb der gleichen Gemeinden. Diese Mängel führen zu Problemen bei Schulwechseln und beeinträchtigen die Effektivität und die Chancengleichheit des Schulsystems. Die unterspezifizierten Lehrpläne lassen offensichtlich auch weitgehend offen, was unter den einzelnen Kompetenzen zu verstehen ist, wie Demeuse und Kollegen/innen mit ihren Interviewdaten eindrücklich demonstrieren. Offenbar bringt Kompetenzorientierung für den Unterricht nur dann Vorteile, wenn der Begriff inhaltlich geklärt und den Lehrpersonen verständlich wird.

Mit dem Wissen (und Können) von Lehrpersonen und der damit verbundenen Wirkung auf deren Unterricht beschäftigt sich der Beitrag von Cauet et al. (2015, in diesem Heft). Diese Autoren fragen nach den Instrumenten, mit denen das für das Unterrichten von Physik relevante Wissen von Lehrpersonen erfasst werden kann, zumal sie in ihrer Untersuchung keine nennenswerten Zusammenhänge zwischen Inhaltswissen (content knowledge) und fachdidaktischem Wissen (beide mit Papier-und-Bleistift gemessen) auf der einen Seite und dem Lerngewinn der Schüler/innen auf der andern Seite nachweisen können. Kritisch anzumerken ist allerdings, dass die Lerngewinne der untersuchten Schüler/innen (aus nur 23 Klassen) insgesamt bescheiden waren (in mehreren Klassen war der Lerngewinn statistisch nicht signifikant). Dadurch stand wenig Varianz zur Verfügung, die die Prädiktoren hätten aufklären können. Bezüglich des fachdidaktischen Wissens wäre immerhin denkbar, dass dessen Messung mittels Vignetten die Lernzugewinne besser vorhersagen könnte (vgl. Brovelli et al., 2014).

Die Varia-Artikel dieses Hefts widmen sich wie immer diversen Themen der Bildungsforschung: Bonvin und Gaudreau (2015, in diesem Heft) stellen anhand einer schriftlichen Befragung angehender Lehrpersonen in der Romandie und in Québec fest, dass sich diese Studierenden im Allgemeinen kompetent fühlen, eine Klasse zu führen, jedoch die eigenen Fähigkeiten bezüglich dem Umgang mit einzelnen Verhaltensproblemen (der Schüler/innen) teilweise weit kriti- 
scher einschätzen. Alexandre Buysse (2015, in diesem Heft) arbeitet in seinem theoretischen, mikrogenetisch ausgerichteten Artikel einzelne Struktur- und Kontrollprozesse heraus, die das Lernen (auch das schulische) regulieren. Petko und Egger (2015, in diesem Heft) schliesslich haben anhand einer grösseren Fragebogenerhebung untersucht, wer eigentlich den Schweizerischen Bildungsserver kennt (und ggf. auch nutzt) und zeigen, dass insbesondere die Gratisressourcen (Lernplattform, Unterrichtsressourcen zum Download) bekannt sind und (wenn auch nicht in ihrem vollen Funktionsumfang) genutzt werden.

\section{Literatur}

Brovelli, D., Bölsterli, K., Rehm, M. \& Wilhelm, M. (2014). Using vignette testing to measure student science teachers' professional competencies. American Journal of Educational Research, 2, 555-558.

Haan, G. de (2002). Die Kernthemen der Bildung für eine nachhaltige Entwicklung. Zeitschrift für internationale Bildungsforschung und Entwicklungspädagogik, 25, 13-20.

Herzog, W. (2013). Bildungsstandards, eine kritische Einführung. Stuttgart: Kohlhammer.

Reichenbach, R. (2014). Ein Interview - Roland Reichenbach im Gespräch. Babylonia, 3/14, 24-29.

Schütte, M., Wirth, J. \& Leutner, D. (2010). Selbstregulationskompetenz beim Lernen aus Sachtexten. Entwicklung und Evaluation eines Kompetenzstrukturmodells. Projekt Selbstregulationskompetenz. Zeitschrift für Pädagogik, 56, 249-257. 